\title{
An improving control strategy for grid -connected current in weak grid
}

\author{
Deng Songyuan ${ }^{1, *}$, Zhao Xia ${ }^{2}$, \\ ${ }^{1}$ School of Automation and Electrical Engineering Lanzhou Jiaotong University, Lanzhou, China \\ ${ }^{2}$ School of Automation and Electrical Engineering Lanzhou Jiaotong University, Lanzhou, China
}

\begin{abstract}
The traditional proportional feedforward control strategy can suppress the low order harmonics in the strong power grid, but in the weak power grid environment, the grid impedance will have an adverse impact on the stability of the system, resulting in the proportional feedforward control can not suppress the low order harmonics well. In order to solve this problem, a control strategy combining resonant feedforward and new repetitive proportional controller is proposed. Firstly, the stability of traditional proportional feedforward and resonant feedforward systems is analyzed and compared by impedance analysis method. Then, a new repetitive controller is added to suppress low order harmonics based on resonant feedforward control, and the stability of single-phase LCL grid connected inverter system is analyzed Matlab / Simulink is used to simulate and verify the system. The results show that the system stability and the ability to suppress low order harmonics are significantly improved.
\end{abstract}

\section{Introduction}

With the rapid development of distributed generation, it brings economic benefits to the society, but also brings many problems, such as the background voltage harmonic oscillation caused by long-distance transmission lines[1]. Due to the dispersion of distributed energy, there are many long distance power transmission cables in the network, and the distributed capacitance of the cables is often large [2]. The distributed capacitance and power factor correction capacitor in the network will resonate with the line inductance of the transmission line, which will increase the total harmonic distortion of the incoming current. LCL grid connected inverter is a third-order system with fixed resonance peak. In order to suppress the resonance peak, capacitor current feedback active feedback control is often used [3]. In order to reduce the total harmonic distortion of incoming current, proportional feedforward control and quasi proportional PR controller are often used to reduce the steady-state error. However, for the multi frequency harmonics or voltage background harmonics generated by inverter dead time, it is necessary to parallel multiple harmonic compensators on the basis of PR controller. However, the design is complex and the system needs to restrain the LCL resonance peak, which leads to the conservative parameter selection. In the weak current network, the traditional proportional feedforward control is coupled with the capacitive current feedback control, which worsens the quality of the incoming current. In order to ensure the robustness and stability of the system, it must be optimized. In reference [4], the influence of grid impedance on grid connected inverter stability is analyzed by Bode diagram, and a control scheme of series complex filter in grid connected voltage proportional feedforward link is proposed. In reference [5], a bandpass filter is proposed to improve the voltage feedforward control, and a multiple harmonic compensator is paralleled in the PR controller to reduce the lower harmonics. In reference [6], a full feedforward control of grid voltage is proposed, which can effectively suppress the influence of grid voltage background harmonics on grid connected current. Taking the singlephase LCL grid connected inverter as the research object, this paper proposes a control strategy combining resonant feedforward and new repetitive control controller, which improves the adaptability to the weak grid and suppresses the current harmonics. Finally, the effectiveness of the proposed strategy is verified by Matlab / Simulink simulation platform.

\section{LCL output impedance model}

Figure 1 shows the model of single-phase LCL grid connected inverter under digital control, in which the DC voltage $u_{\text {in }}$ and the input current $u_{\text {inv }} . L_{1}, C$ and $L_{2}$ make up LCL filter [7]. $H_{\mathrm{i} 1}$ and $H_{\mathrm{i} 2}$ are the sampling coefficients of capacitive current loop and grid connected current loop $u_{\mathrm{pcc}}$ extracts the voltage phase through PLL and multiplies the reference current amplitude I* generated by outer loop to obtain the reference grid current $i_{2}{ }^{*} . i_{2}{ }^{*}$ is subtracted from the sampled grid current $i_{2}$ and enters the current regulator $G_{\mathrm{i}}(\mathrm{s})$. In order to suppress the influence of the background harmonic, the feed-forward controller $G_{\mathrm{f}}(\mathrm{s})$

\footnotetext{
* Corresponding author: 460135365@qq.com
} 
is added. The output current of $G_{\mathrm{i}}(\mathrm{s})$ is added with the output signal of $G_{\mathrm{f}}(\mathrm{s})$, and the sampling current of capacitor is subtracted to get PWM modulation wave $u_{\mathrm{m}}$.
The modulation wave is sent into the PWM generator, and the duty cycle is generated after pulse width modulation to control the inverter switch.

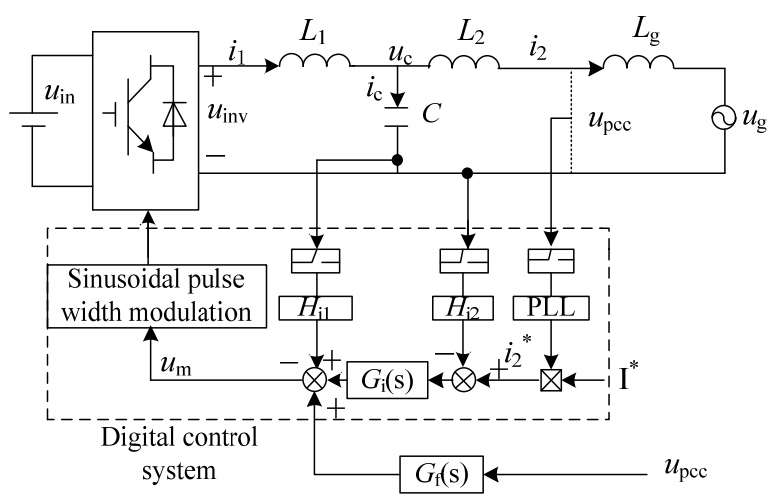

Figure. 1. LCL single phase grid connected inverter model

According to figure 1, the system control block diagram is drawn without considering the system control delay, as shown in Figure 2.

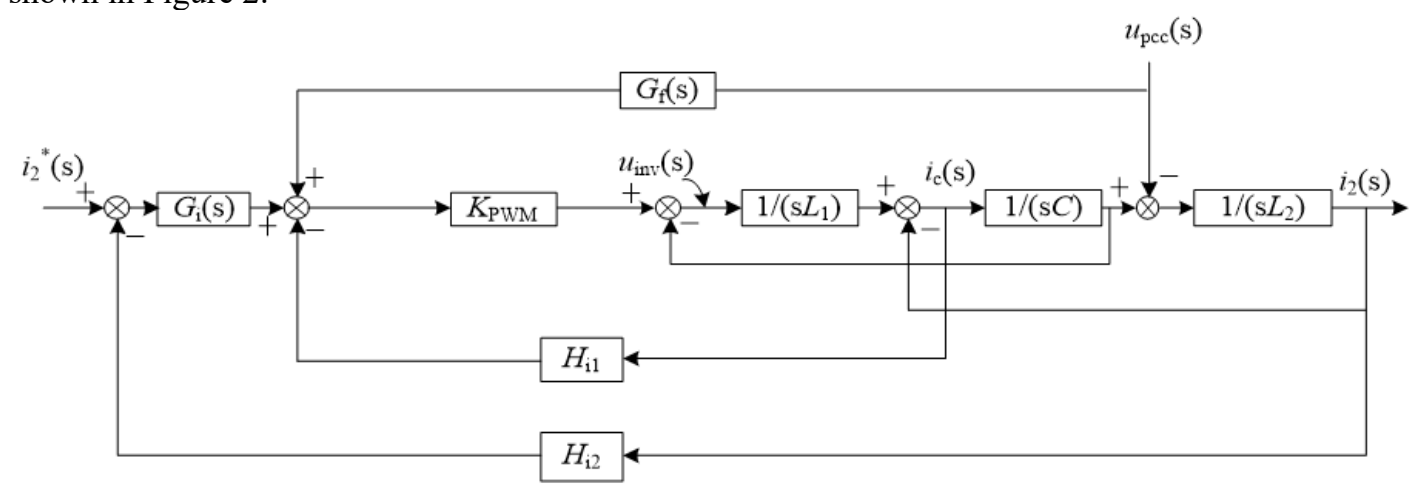

Figure. 2. Mathematical model of LCL grid connected inverter

Figure 3 is obtained by simplifying the control block diagram.

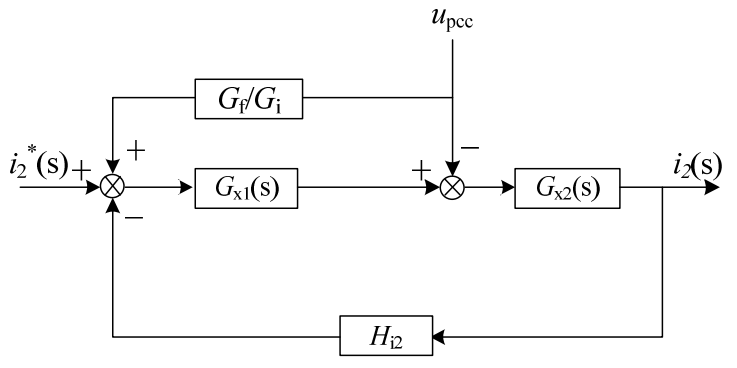

Fig. 3. Control block diagram after transformation

Writing the expressions of $G_{\mathrm{X} 1}(\mathrm{~s})$ and $G_{\mathrm{X} 2}(\mathrm{~s})$ according to figure 3 .

$$
\begin{gathered}
G_{x 1}(s)=\frac{K_{P W M} G_{i}(s)}{s^{2} L_{1} C+s C H_{i 1} K_{P W M}+1} \\
G_{x 2}(s)=\frac{s^{2} L_{1} C+s C H_{i 1} K_{P W M}+1}{s^{3} L_{1} L_{2} C+s^{2} L_{2} C H_{i 1} K_{P W M}+s\left(L_{1}+L_{2}\right)}
\end{gathered}
$$

Let $h=G_{x 1}(s) G_{x 2}(s)$ get the expression of grid connected current in formula (3).

$$
i_{2}(s)=\frac{h}{1+H_{i 2} h} i_{2}^{*}-\frac{G_{x 2}(s) G_{i}(s)-G_{f}(s) h}{\left(1+H_{i 2} G_{x 1}(s) G_{x 2}(s)\right) G_{i}(s)} u_{p c c}
$$

From the expression of equation (3), we can get the equivalent circuit of Norton, as shown in Figure 4.

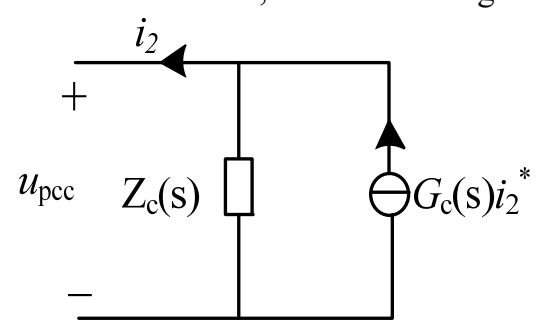

Figure. 4. Norton equivalent circuit

Under these circumstances:

$$
\begin{gathered}
i_{g}=\frac{Z_{c}}{Z_{c}+Z_{g}} G_{c} i_{2}^{*}-\frac{1}{Z_{c}+Z_{g}} u_{g} \\
G_{c}=\frac{G_{x 1}(s) G_{x 2}(s)}{1+H_{i 2} G_{x 1}(s) G_{x 2}(s)} \\
Z_{c}(s)=\frac{\left(1+H_{i 2} G_{x 1}(s) G_{x 2}(s)\right) G_{i}(s)}{G_{x 2}(s) G_{i}(s)-G_{f}(s) G_{x 1}(s) G_{x 2}(s)} \\
Z_{c}(s)=\frac{s^{3} L_{1} L_{2} C+s^{2} L_{2} C H_{i 1} K_{p w m}+s\left(L_{1}+L_{2}\right)+G_{i}(s) H_{i 2} K_{p u m}}{s^{2} L_{1} C+s C H_{i 1} K_{p u m}+1-G_{f}(s) K_{p u m}}
\end{gathered}
$$


$Z_{\mathrm{C}}$ is the output impedance of the inverter. It can be seen from Figure 4 that the grid current is affected by the voltage of the common coupling point, and the common coupling point also contains the grid impedance information under the weak current network. Because the resistance is favorable to the stability of the system, the net impedance is obtained from the pure sensitivity here. So $u_{p c c}=s L_{g} i_{g}+u_{g}$.

\section{System robustness analysis}

The current regulator GI (s) adopts quasi PR controller here, and the transfer function of quasi PR controller is formula (8).

$$
G_{i}(s)=K_{p}+\frac{2 K_{r} \omega_{c} s}{s^{2}+2 \omega_{c} s+\omega_{0}^{2}}
$$

Next, impedance analysis method is used to analyze the robustness of resonant feedforward and proportional feedforward [8].

The transfer function of proportional feedforward is equation 9 .

$$
G_{f 1}=k
$$

The transfer function of resonant feedforward is equation 10

$$
G_{\mathrm{f} 2}=\frac{k_{r} w_{0} s}{s^{2}+k_{r} w_{0} s+w_{0}^{2}}
$$

Bode diagrams of proportional feedforward + PR control and resonant feedforward + PR control are drawn respectively. As shown in figures 5 and 6 .
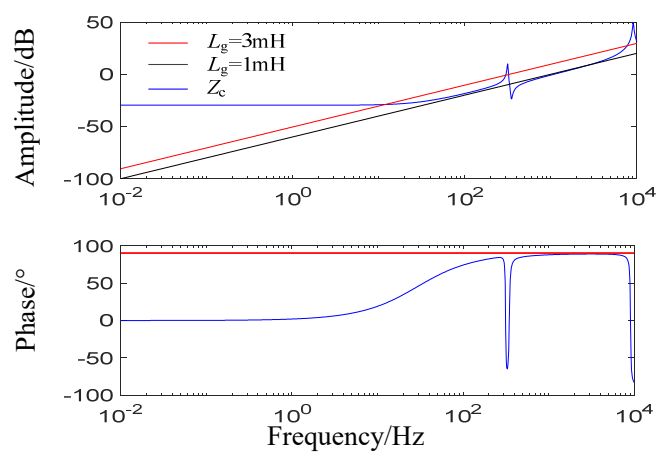

Figure. 5. Bode diagram of proportional feedforward + PR control
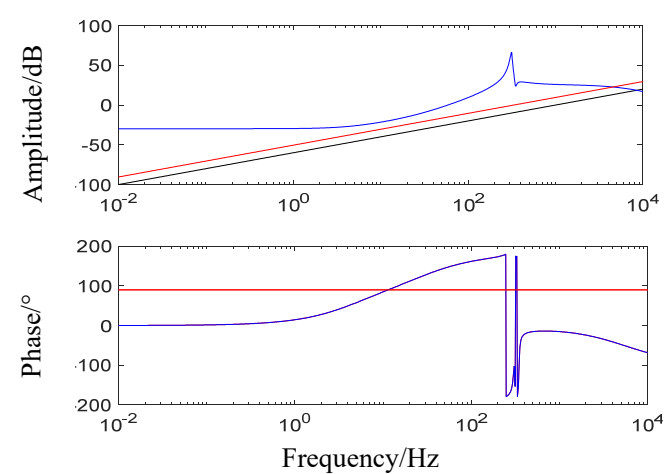

Figure. 6. Bode diagram of resonance feedforward $+P R$ control
It can be seen from the figure that when the system adopts $\mathrm{PR}+$ proportional feedforward in the weak current network, the impedance junction point is at the resonance peak, which will cause system oscillation and worsen the quality of incoming current. When PR + resonant feedforward control is adopted, the system's junction point moves to the right, and the phase difference of the junction point is within 180 degrees. so the system is stable and has a large stability margin. In conclusion, $\mathrm{PR}+$ resonance feedforward control improves the adaptability of the system to the inductive reactance of weak current network.

\section{New repetitive control strategy}

Due to the weak ability of the system to suppress the low order background harmonics, the parallel multiple harmonic compensator is generally used to reduce the low order harmonics, but it will reduce the phase margin of the system and make the design more complex. Therefore, a scholar proposed a new repetitive proportional compound controller. This controller can provide high gain at low frequency and gain attenuation at high frequency, which can not only track the fundamental signal without error, but also reduce low order harmonics [9]. This paper uses its method for reference and applies it to LCL grid connected inverter. Its structure is shown in Figure 7.

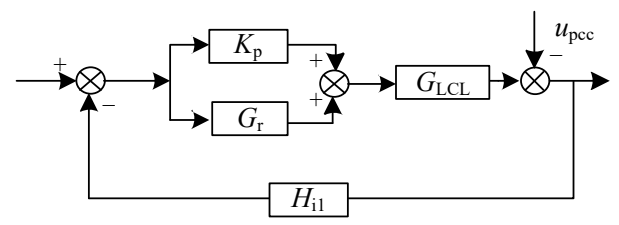

Figure. 7. Block diagram of repetitive proportional controller

$$
G_{r}(z)=\frac{Q(z) z^{-N}}{1-Q(z) z^{-N}} z^{m} k_{r} S(z)
$$

In equation $11, Q(\mathrm{z})$ is $\mathrm{RC}$ internal model filter, which is used to improve the stability and robustness of the system; $\mathrm{N}$ is the number of delay beats in each cycle, and $\mathrm{N}$ is the ratio of sampling frequency to $i_{\text {ref }}$ fundamental frequency of reference signal; $z^{\mathrm{m}}$ is phase lead compensation; $S(\mathrm{z})$ is compensator.

The impedance analysis method is used to analyze the stability of the system with the new repetitive controller, as shown in Figure 8. 

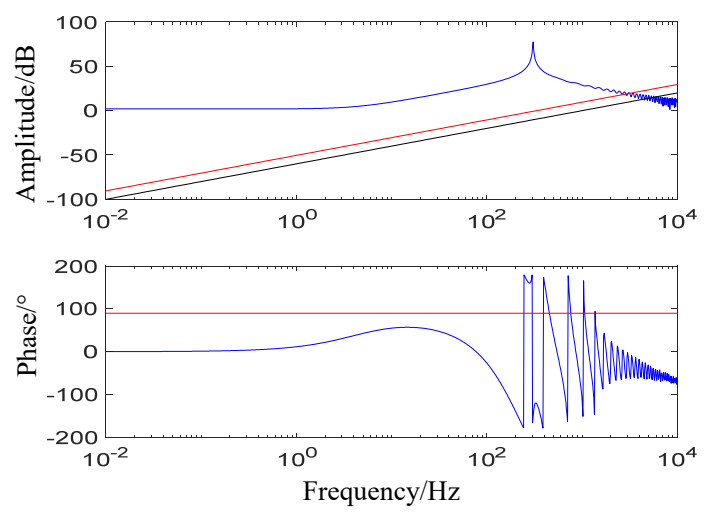

Figure. 8. Bode diagram of resonant feedforward + New Repetitive Controller

Figure 8 is the impedance Bode diagram of the new repetitive controller added on the basis of resonance feedforward. It can be seen that the system has good stability and high robustness, and the high gain at the low order harmonics will suppress the low order harmonics, thus optimizing the quality of grid connected current.

\section{Smulation verification}

In order to verify the effectiveness of the proposed method, the LCL grid connected inverter model as shown in Figure 1 is built by Matlab/Simulink simulation software, and the parameters are shown in Table 1.

Table 1. System parameters

\begin{tabular}{|c|c|c|c|}
\hline $\begin{array}{c}\text { Parameters } \\
\text { and units }\end{array}$ & $\begin{array}{c}\text { Numerical } \\
\text { value }\end{array}$ & Parameters and units & $\begin{array}{c}\text { Numerica } \\
1 \text { value }\end{array}$ \\
\hline $\begin{array}{c}\text { DC Bus } \\
\text { Voltage } \\
u_{\mathrm{dc}} / \mathrm{V}\end{array}$ & 380 & $\begin{array}{l}\text { Filter capacitor } \\
C / \mu \mathrm{F}\end{array}$ & 10 \\
\hline $\begin{array}{c}\text { Grid voltage } \\
u_{\mathrm{g}} / \mathrm{V}\end{array}$ & 220 & $\begin{array}{c}\text { Grid side inductance } \\
L_{2} / \mu \mathrm{H}\end{array}$ & 150 \\
\hline $\begin{array}{c}\text { Fundamental } \\
\text { frequency } \\
f_{0} / \mathrm{Hz}\end{array}$ & 50 & $\begin{array}{c}\text { Proportional } \\
\text { coefficient of PR } \\
\text { controller } K_{\mathrm{p}}\end{array}$ & 0.72 \\
\hline $\begin{array}{c}\text { Switching } \\
\text { frequency } \\
f_{\text {sw }} / \mathrm{kHz}\end{array}$ & 10 & $\begin{array}{c}\text { Resonance } \\
\text { coefficient of PR } \\
\text { controller } K_{\mathrm{r}}\end{array}$ & 70 \\
\hline $\begin{array}{c}\text { Sampling } \\
\text { frequency } \\
f_{\mathrm{s}} / \mathrm{kHz}\end{array}$ & 20 & $\begin{array}{c}\text { Feedback coefficient } \\
\text { of grid connected } \\
\text { current } H_{\mathrm{i} 2}\end{array}$ & 0.12 \\
\hline $\begin{array}{c}\text { Inverter side } \\
\text { inductance } \\
L_{1} / \mu \mathrm{H}\end{array}$ & 500 & $\begin{array}{c}\text { Capacitive current } \\
\text { feedback coefficient } \\
H_{\mathrm{i} 1}\end{array}$ & 0.15 \\
\hline
\end{tabular}

Figure 9 and Figure 10 respectively show the simulation results of proportional feedforward $+\mathrm{PR}$ control strategy and resonant feedforward+New Repetitive Control Strategy under strong power grid. It can be seen that THD of proportional feedforward + PR control strategy is $1.38 \%$, and THD of resonant feedforward + new repetitive control strategy is $1.11 \%$. Both of them meet the grid connection standard.
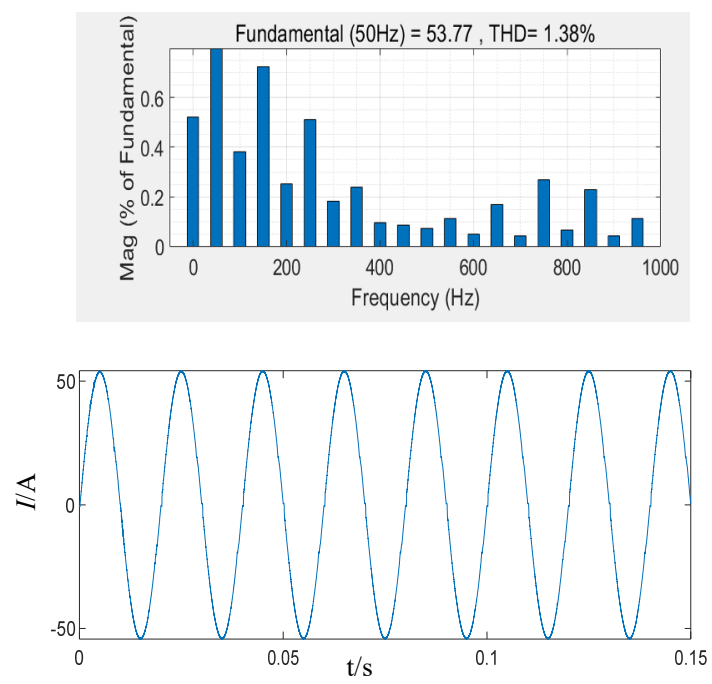

Figure. 9. Waveform and spectrum of proportional feedforward + PR grid connected current

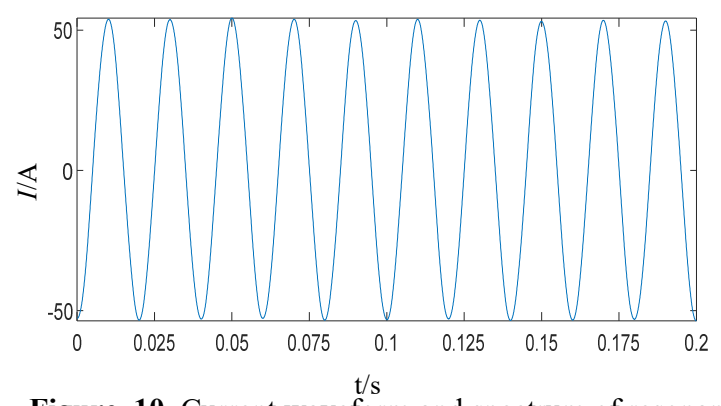

Figure. 10. Current waveform and spectrum of resonant feedforward + PR grid connection

Keeping the parameters unchanged, the two control modes are respectively placed in the system with $1 \mathrm{mH}$ grid impedance. It can be seen from Figure 11 that when proportional feed-forward + PR control mode is adopted, the system will vibrate, and the total harmonic distortion (THD) of incoming current is $8.83 \%$, exceeding the international standard. It shows that this control method has poor robustness, poor adaptability to the inductive reactance of power grid, and contains high low order harmonics. It can be seen from Fig. 12 that the total harmonic distortion of the incoming current is $1.22 \%$, which meets the grid connection standard. It shows that the system has a strong adaptability to the inductive reactance of power grid, and has a significant effect on the suppression of low order harmonics, and has a significant effect on the attenuation of high frequency harmonics. 

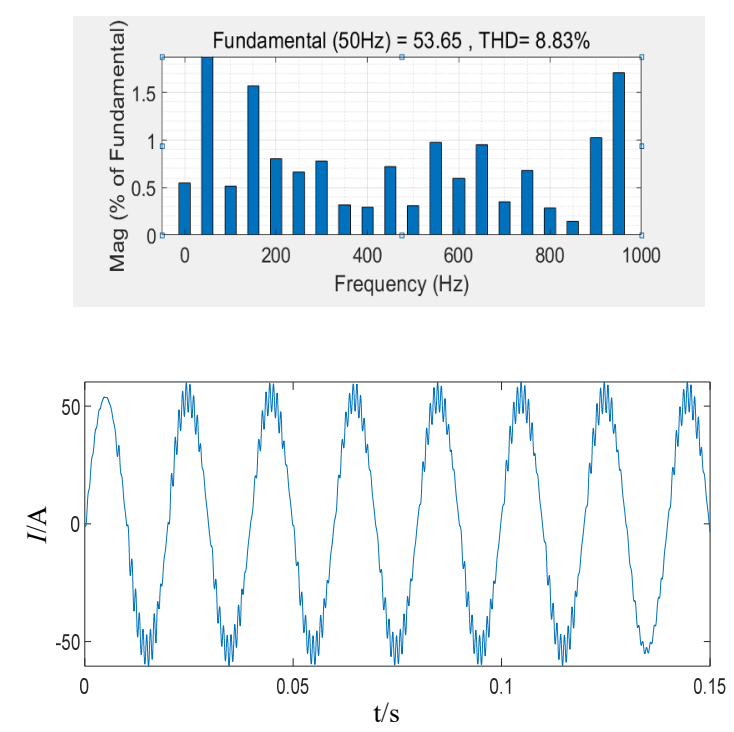

Figure. 11. Current waveform and spectrum of proportional feedforward + PR grid connection when $L_{\mathrm{g}}=1 \mathrm{mH}$
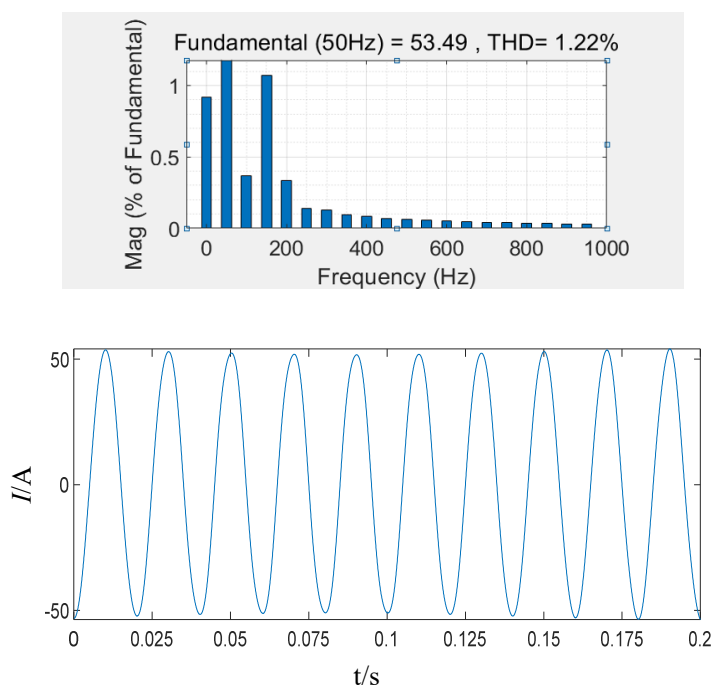

Figure. 12. Current waveform and spectrum of resonant feedforward + new repetitive control grid connection when $\mathrm{Lg}$ $=1 \mathrm{mH}$

\section{Conclusion}

The traditional proportional feedforward+PR control will couple with the capacitor current active damping feedback circuit in the weak current network, which will seriously deteriorate the quality of the incoming current, and the suppression of low order harmonics is weak. In order to suppress the low order harmonics, the phase margin of the system will be reduced by using the parallel multiple harmonic controller, and the design is more complex.

The control strategy of resonant feedforward combined with new repetitive control proposed in this paper can not only improve the adaptability to weak grid inductance, but also reduce the low order harmonics of grid connected current, so it can optimize the grid connected current quality of inverter under weak current.

\section{Acknowledgments}

Supported by National Natural Science Foundation of China (51767015);

Supported by Gansu Natural Science Foundation of China (2016gs07210);

\section{References}

1. Sun, X, Zhang, M, Wang, $H$ et al. (2019) Background voltage harmonic suppression strategy of a distribution network. In:2019 22nd International Conference on Electrical Machines and Systems (ICEMS). Harbin, China. pp. 1-5.

2. Sun, J. (2011) Impedance-based stability criterion for gridconnected inverters. IEEE Transactions on Power Electronics, 26(11): 3075-3078.

3. Ling, Z., Xu, C. B, Cao, C. C, et al. (2020) Novel single current feedback control scheme for gridconnected inverter with LCL filters. Electrical Measurement and Instrumentation, 57(02): 135$140+152$.

4. Zheng, Z, Huang, X, Yang, M, et al. (2018) Grid voltage feedforward control strategy for weak grid based on complex filter. Power System Protection and Control, 46(24):70-75.

5. Yang, L. Y, Guo, R, Zhang, et al. (2020) Improvement strategy for grid-connected current quality of an inverter under non-ideal grid conditions. Power System Protection and Control, 48(15): 10-18.

6. Chai, X. H, Zhang, C. J, Zhao, X. J, et al. (2020) Research on power grid background harmonic suppression based on grid voltage full-feedforward. Power Electronics, 54(08): 5-7+18.

7. Wang, L, Sun, P. J, Xue, T. Y, et al. (2020) A delay compensation method to improve the current control performance of the LCL-type grid-connected inverter. Proceedings of the CSEE, 40(19): 63206330.

8. Zhang, Z. J, Li, J. W, Dong, Y, et al. (2020) Method of resonance instability analysis of multiple gridconnected inverters in weak grid. Electrical Engineering, 21(10): 21-28.

9. Zhao, Q. S, Chen, S. S, Zhou, X. Y, et al. (2019) Analysis and design of combination controller based on repetitive control and proportional control for harmonics suppression of grid-tied inverters. Transactions of China Electrotechnical Society, 34(24): 5189-5198. 